\title{
Evaluation and optimization of seal behaviour through solid contamination of heat-sealed films
}

\author{
Bram Bamps $^{1}$ (I) | Karlien D'huys ${ }^{2}$ | Ina Schreib ${ }^{3}$ | Benjamin Stephan ${ }^{3}$ | \\ Bart De Ketelaere $^{2}$ । Roos Peeters ${ }^{1}$
}

\author{
${ }^{1}$ Hasselt university, IMO-IMOMEC, Packaging \\ Technology Center, Wetenschapspark 27, \\ 3590 Diepenbeek, Belgium \\ ${ }^{2}$ Department of Biosystems, MeBioS, KU \\ Leuven, Kasteelpark Arenberg 30, B-3001 \\ Heverlee, Belgium \\ ${ }^{3}$ Division Processing Technology, Fraunhofer \\ Institute for Process Engineering and \\ Packaging, Heidelberger Str. 20, 01189 \\ Dresden, Germany \\ Correspondence \\ Bram Bamps, Hasselt university, IMO- \\ IMOMEC, Packaging Technology Center, \\ Hasselt 3500, Belgium. \\ Email: bram.bamps@uhasselt.be \\ Funding information \\ German Federal Ministry for Economic Affairs \\ and Energy, Grant/Award Number: 172 EBR; \\ Agentschap Innoveren \& Ondernemen, Grant/ \\ Award Number: 150817
}

A method is presented to apply solid powder/granulate contamination (ground coffee and blood powder) in between the heat conductive seals of flexible packaging materials. A response surface method is tested and validated to optimize seal strength of heat conductive sealing with and without solid contamination. In this study, a maximal seal strength is defined as optimal. Using these methods, three typical packaging films with varying seal layer composition (metallocene linear low-density polyethylene (LLDPE), plastomer, and sodium ionomer) are maximized towards contaminated seal strength. Contamination caused a decrease in seal strength and narrowed down the process window (seal temperature and time combinations) in which at least $90 \%$ of the maximal strength is obtained. The influence of seal layer composition on the clean and solid (ground coffee and blood powder) contaminated seal performance (seal strength, process window, and leak tightness) was evaluated. The film with the plastomer-based seal layer outperformed the other films with respect to the width of the process window. It also reached a higher seal strength and a higher amount of leak tight seals (evaluated with the dye penetration test) after optimization. The hot tack test was evaluated as predictive test for the contaminated seal strength. The results of this study do not support an indicative relationship.

\section{KEYWORDS}

hot tack, polyethylene, process window, response surface model, seal through contamination

\section{1 | INTRODUCTION}

The closure of flexible packages is mainly established by applying heat sealable materials at the inner side of the packaging material, which creates a seal after bringing the inner sides together, heating, and compressing them. The materials melt, and polymer chains at the interface entangle, and after cooling, a strong seal can be obtained. Besides chain entanglement, intermolecular and mechanical bonds are possible, but these adhesion mechanisms are less common for heat seals. There are several technologies to heat a seal, such as heat conductivity, inductivity, and ultrasonic vibrations. Conductive heating is the most popular technology for flexible packaging. ${ }^{1}$ Industrial research in the past showed that one-third of sealed packages are of an insufficient quality. ${ }^{2,3}$ Therefore, the tightness of the package, which is in general essential to prevent microbial and biochemical degradation of food, cannot be guaranteed. For $65 \%$ of the packages that show seal defects, contamination in between the seal layers is the major cause. ${ }^{3}$ Only $16 \%$ of the packers perform an

This is an open access article under the terms of the Creative Commons Attribution-NonCommercial-NoDerivs License, which permits use and distribution in any medium, provided the original work is properly cited, the use is non-commercial and no modifications or adaptations are made.

(C) 2019 The Authors. Packaging Technology and Science Published by John Wiley \& Sons Ltd 
inspection of all produced goods. The majority just inspects samples with an interval of 30 minutes. $^{3}$

One way to prevent seal defects as a result of contamination is to work with materials that are able to seal through contamination at particular seal settings to decrease the amount of defective packages. Several polyethylene (PE)-based packaging materials have been developed with a good seal performance through contamination in the last decades. Examples of these materials are metallocenecatalysed linear low-density PE (LLDPE), polyolefin plastomer, and ionomer. $^{4}$

In scientific and technical papers, several tests are performed on packaging films with contaminated seals such as seal strength, leak rate, and degree of particle encapsulation (caulkability). ${ }^{5-9}$ This study is focussed on seal strength. Hot tack tests are performed to evaluate the resistance of packaging films against spring back forces. $^{6,7,9,10}$ The relation of seal through contamination and hot tack performance is part of this study. In the last decades, the influence of seal material composition on the contaminated seal performance was the topic of a limited amount of studies, and these studies did not include a well-described application method for solid contamination..$^{5,6,8,9}$ Moreover, there are no methods described in these studies to obtain the optimal or maximal seal performance through contamination.

\section{2 | OBJECTIVES}

The main objective of this study is to present a method to optimize the granular-contaminated seal strength of packaging films. Firstly, a protocol to apply solid contamination in a standardized way is described, this protocol was missing in previous studies with powder or granulate contamination. Secondly, an optimization method is presented that is based on a previous study on ultrasonic sealing. ${ }^{11}$ In this study, a similar methodology is used in order to receive information on the influence of all relevant seal parameters on the heat conductive sealing process based on a limited number of carefully selected experiments.

A second objective is to evaluate the influence of variation in seal layer composition (metallocene $\mathrm{PE}$, plastomer, and ionomer) on the seal through contamination performance (seal strength, width process window, and leak tightness) by using the application and optimization methods of this study. Hot tack tests are evaluated as predictive tests for contaminated seal performance.

\section{3 | MATERIALS AND METHODS}

\section{1 | Materials}

\subsection{1 | Commercial multilayer packaging films for flowpack applications}

Table 1 shows the multilayer structure of three flowpack films, evaluated and optimized in this study. Each film has a 12- $\mu \mathrm{m}$ thick PE terephtalate (PET) outer layer. A 3-layer blown film line with three nozzles is used for the production of the seal layers. The upper $35 \mu \mathrm{m}$ has the same composition for the three films, containing a blend of low-density PE (LDPE) and metallocene LLDPE (mLLDPE). The films differ mainly in the $15-\mu m$ lower seal layer. Film 1 has a blend of LDPE and mLLDPE while film 2 has a blend of LDPE and polyolefin plastomer (mLLDPE with a high amount of comonomer) in that area. Both films have $2 \%$ processing aid in the lower seal layer. Film 3 has a $5-\mu m$ layer of acid copolymer resin between the $35-\mu \mathrm{m}$ $\mathrm{PE}$ and the $10-\mu \mathrm{m}$ ionomer layer to ensure the bonding of both layers.

\subsection{2 | Contamination types}

Two types of solid contamination are used in this study: (a) sieved ground coffee (Delhaize, Belgium; sieved to obtain a particle size between 500 and $630 \mu \mathrm{m}$ using a Fritsch analysette 3 sieve shaker system) and (b) freeze-dried pork blood powder (Solina, Germany; particles with an average size of $100 \mu \mathrm{m})$.

\section{2 | Methods}

\subsection{1 | Sample preparation contaminated seals}

Films, sealed samples, and solid contamination are stored at a temperature of $23^{\circ} \mathrm{C}$ and a relative humidity of $50 \%$. The precision balance OHAUS Explorer (Mettler-Toledo International Inc, USA) with readability of $0.0001 \mathrm{~g}$ is used for all weighings. Figure 1 shows an illustration of the sample preparation. The sample is cut in machine direction (MD) with a width of $50 \mathrm{~mm}$ and an appropriate length to do a seal strength test (in this study the length exceeded $100 \mathrm{~mm}$ ) (I). An area of 20 by $40 \mathrm{~mm}$ is then marked on the film sample. It is important that the chosen length has sufficient extra margin compared with the seal length to ensure that the contamination is distributed over the full length of the seal. In this case, $20 \mathrm{~mm}$ is chosen because the used sealer produces $10 \mathrm{~mm}$ length seals. The required amount of solid

TABLE 1 Multilayer structure of the three packaging films

\begin{tabular}{|c|c|c|c|c|c|}
\hline Film 1 & & Film 2 & & Film 3 & \\
\hline PET & $12 \mu \mathrm{m}$ & PET & $12 \mu \mathrm{m}$ & PET & $12 \mu \mathrm{m}$ \\
\hline 80\% LDPE-20\% mLLDPE & $15 \mu \mathrm{m}$ & 80\% LDPE-20\% mLLDPE & $15 \mu \mathrm{m}$ & 80\% LDPE-20\% mLLDPE & $35 \mu \mathrm{m}$ \\
\hline 68\% LDPE-30\% mLLDPE & $15 \mu \mathrm{m}$ & 68\% LDPE-30\% plastomer & $15 \mu \mathrm{m}$ & Sodium ionomer & $10 \mu \mathrm{m}$ \\
\hline
\end{tabular}

Abbreviations: LDPE, low-density polyethyelene; mLLDPE, metallocene linear low-density polyethylene; PET, polyethylene terephtalate. 


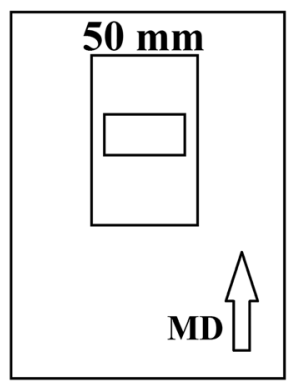

(I)

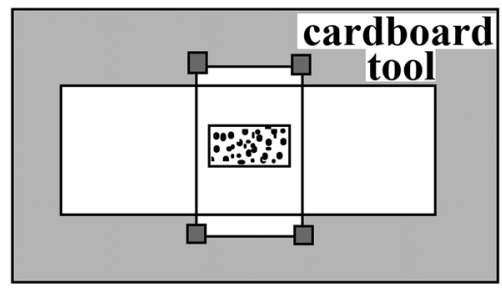

(II)

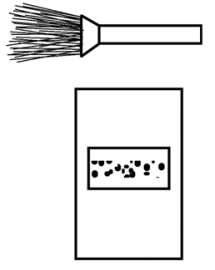

(III)
FIGURE 1 Contaminated seal preparation

contamination is weighed. In order to facilitate the sealing with contamination, a simple cardboard tool is cut out of a cardboard sheet with an inner hole with larger width and length than the seal jaw. After one film sample is fixed with plastic tape to this cardboard tool, the contamination is applied with a small spoon in the designated area. In this study, $0.020 \mathrm{~g}$ is applied in a $20 \times 40-\mathrm{mm}^{2}$ region to achieve a $25 \mathrm{~g} \mathrm{~m}^{-2}$ contamination density. When the contamination is applied and evenly distributed by eye, the second film sample is used to cover the contamination and fixed with plastic tape (II). The cardboard tool with contaminated film samples is manually placed between the seal jaws, and the seal is formed. Seals are produced with the Labthink HST-H3 heat seal tester (Labthink Instruments Co Ltd, People's Republic of China). This sealer has two flat aluminium jaws covered with silicon tape to prevent the contamination from sticking to the jaws. After sealing, the amount of contamination, which is not trapped within the seal area, is carefully removed using a small brush (III).

\subsection{2 | Film characterization}

\section{Differential scanning calorimetry}

To characterize the thermal behaviour of the packaging material and to relate this to the heat conductive sealing performance, differential scanning calorimetry (DSC) measurements were executed with the instrument 2920 MDSC V.2.6A (TA instruments, USA). The three film samples and the main components of the seal layer (granulate form of LDPE, mLLDPE, plastomer, acid copolymer resin, and sodium ionomer) were tested in a sequence of two controlled heatings and one cooling down stage within the range of $10^{\circ} \mathrm{C} \rightarrow 200^{\circ} \mathrm{C}$ at a heating/cooling speed of $10^{\circ} \mathrm{C} \mathrm{min}{ }^{-1}$. The heating does not exceed $200^{\circ} \mathrm{C}$ to prevent the PET layer from melting as this study focusses on the components of the seal layer and the seal layer as its whole. The first heating cycle is performed to delete the thermal history. The second heating cycle is used to obtain the melting peak temperature and the melting onset temperature (intersection of the tangent of the peak and the extrapolated baseline). Both of these temperatures are used to compare the materials.

\section{Hot tack}

Twenty-five-millimetre-wide samples were tested with a J\&B Hot Tack Tester model 5000 MB (Vived-Management, Belgium) according to ASTM F1921 at a tensile speed of $200 \mathrm{~mm} \mathrm{~s}^{-1}$. Maximum force is divided by seal width $(25 \mathrm{~mm})$ to obtain the hot tack strength. Seal time, seal pressure, and cooling time were kept constant at respective values of 1.0 second, $0.3 \mathrm{~N} \mathrm{~mm}^{-2}$, and 0.1 second while seal temperature was varied. Samples are tested in threefold, and average values and standard deviations are shown.

\subsection{3 | Seal characterization}

Seal strength is tested according to ASTM F88 on 15-mm wide samples. These samples are tested unsupported. Clamp distance is $10 \mathrm{~mm}$, and tensile speed is $300 \mathrm{~mm} \mathrm{~min}{ }^{-1}$. The seal strength is obtained by dividing maximum force with seal width ( $15 \mathrm{~mm})$.

The dye penetration test uses an aqueous solution with $0.05 \%$ indicator dye (toluidine blue) and 3\% wetting agent (PE glycol octylphenyl ether) to determine if there are leaks. It allows to detect and locate channel leaks, which are equal to or greater than channels caused by a wire with diameter of $50 \mu \mathrm{m}$. The qualitative information (leak or no leak) delivers complementary information to seal strength. It is performed according to ASTM-F3039 on samples as shown in Figure 2. The edges of the (contaminated) seal samples need to be sealed to have a reservoir where 1-mL dye can be poured in (I). After removing the sealed edges at one side of the seal, the seal can be pressed to an absorbing white paper after adding the dye. The package is held in a vertical position for 1 minute so that the dye can penetrate through possible channel leaks by gravity (II). If a stain is visible on the paper, the seal is reported as leaker. This test can be done prior to performing the seal strength test if the seal strength of a sample is

\section{Contaminated seal}

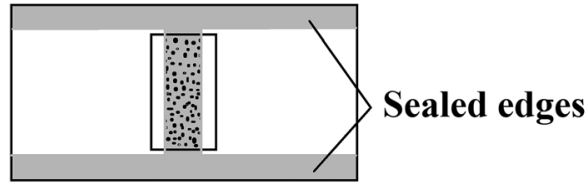

(I)

FIGURE 2 Dye penetration test

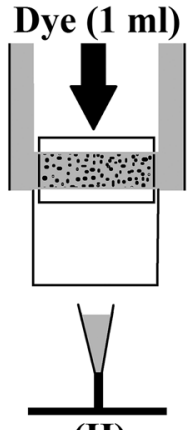

(II) 
not influenced by the penetrating dye. This was confirmed by preliminary tests (results not shown).

Samples that pass this dye penetration test are considered as leak tight.

\subsection{4 | Maximization of contaminated seal quality}

In order to assess the effect of the sealing parameters (temperature, time, and pressure) on the seal quality of both clean and contaminated (coffee and blood powder) seals, the approach presented in D'huys et al was followed. This approach is based on the concepts of design of experiments (DOE) and response surface modelling. The steps will be briefly described below for the case considered in this study.

First, a design space was defined that includes the three most important seal parameters: jaw temperature $\left({ }^{\circ} \mathrm{C}\right)$, seal time $(\mathrm{s})$, and seal pressure $\left(\mathrm{N} \mathrm{mm}^{-2}\right)$. The effect of these parameters is studied within certain limits. There are no strict rules to set these limits. They can be based on film specifications, on the limits of the sealing process, on the relevance for the application, and/or on results of preliminary tests. In this study, the limits considered for jaw temperature, seal time, and pressure are, respectively, $120^{\circ} \mathrm{C}$ to $180^{\circ} \mathrm{C}, 0.4$ to $1.0 \mathrm{~s}$, and 1.0 to $4.0 \mathrm{~N} \mathrm{~mm}^{-2}$.

Secondly, an experimental design is set up. In this study, a 20-point I-optimal design was selected, rather than the Box-Behnken design of a previous study. ${ }^{11}$ This I-optimal design allows to include corner points of the design space, which represent extreme parameter combinations. Moreover, it allows to include a third-order effect of seal pressure in the response surface model, which was shown to possibly be of interest based on preliminary experiments.

The third step involves fitting a response surface model with three input variables (temperature, time, and pressure) to the seal strength values obtained at the 20 experimental runs. The following quadratic model with interactions was fitted:

$$
\begin{aligned}
y= & \beta_{0}+\beta_{1} x_{1}+\beta_{2} x_{2}+\beta_{3} x_{3}+\beta_{12} x_{1} x_{2}+\beta_{23} x_{2} x_{3}+\beta_{13} x_{1} x_{3} \\
& +\beta_{11} x_{1}^{2}+\beta_{22} x_{2}^{2}+\beta_{33} x_{3}^{2}+\beta_{333} x_{3}^{3}+\varepsilon
\end{aligned}
$$

where $x_{1}, x_{2}$, and $x_{3}$ are the three input parameters, seal temperature, time, and pressure, $y$ is the seal strength and $\varepsilon$ is the error term. Besides the main effects, the interaction terms and quadratic terms were also considered in the model. Moreover, for pressure, a third-order effect was also included in the model. Nonsignificant effects are removed from the model by an all possible subsets procedure. The model with the best fitting subset of effects is selected. The criteria of this selection are $R^{2}$, Akaike information criterion (AIC), and Bayesian information criterion (BIC). For a more detailed description of the model selection, the reader is referred to the previous study on ultrasonic seals. ${ }^{11}$

In a fourth step, the response surface model was utilized for optimizing the input variables towards the response (seal strength). In this study, maximum seal strength is defined as an optimal result and was thus assigned a desirability $=1$. In addition to determining one optimal parameter setting, a process window can be generated, which for example excludes parameter combinations resulting in seal strength below a certain threshold. In this study, process windows were generated containing only those parameter combinations at which at least $90 \%$ of the maximum seal strength is reached.

In a fifth and last step, the optimum was experimentally validated by performing repeated measurements $(n=10)$ at the optimal settings to check if the predicted optimum lies within the confidence interval calculated from the measured values. The success of the confirmation is assessed by following an approach based on a confidence interval calculation of the confirmation runs (CICon approach) as suggested in previous research. ${ }^{12,13}$ For details, the reader is referred to the previous study on ultrasonic seals. ${ }^{11}$

\section{4 | RESULTS AND DISCUSSION}

\section{1 | Film characterization}

It is not always possible to compare DSC results of packaging films with blown extruded films with $100 \%$ pure material such as plastomer because of low viscous behaviour of this substance. Because of this, the results of the seal layers of the three packaging films are compared with the individual components in granulate form. This allows to identify similarities and to suggest explanations in differences and similarities of the three packaging films. The melting onset and peak temperatures of the films and granulates in this study are shown in Table 2. The values of film 1 are in between the values of its main components LDPE and mLLDPE in granulate form. In a previous study on blended films of LDPE and mLLDPE, it was found that the melting point of the blended monolayer was between the values observed for mLLDPE and LDPE films. ${ }^{14}$ The melting onset temperature of film 2 is decreased with $5^{\circ}$ compared with film 1 . The presence of plastomer instead of mLLDPE in the lower $15 \mu \mathrm{m}$ of the seal layer is suggested as explanation since plastomer has a lower melting point than mLLDPE. This is indicated by melting peak temperatures of the components in granulate form. The melting peak temperature of the seal layer of film 2 is close to the value of film 1. Acid copolymer and sodium ionomer presence,

\begin{tabular}{|c|c|c|c|c|c|c|c|c|}
\hline & \multicolumn{3}{|l|}{ Film } & \multicolumn{5}{|c|}{ Granulate of Film Component } \\
\hline & 1 & 2 & 3 & LDPE & mLLDPE & Plastomer & Acid Copolymer & Sodium lonomer \\
\hline $\mathrm{T}_{\text {melt onset }}\left({ }^{\circ} \mathrm{C}\right)$ & 100 & 95 & 98 & 102 & 95 & 87 & 77 & 70 \\
\hline
\end{tabular}

TABLE 2 Melting onset and melting peak temperatures of films and granulates, tested with DSC

Abbreviations: DSC, differential scanning calorimetry; mLLD, metallocene linear low density; LDP, low-density polyethyelene. 
which have lower melting points than LDPE, mLLDPE, and plastomer, does not decrease the melting peak and onset temperature of film 3. These components are present in low amount in proportion to LDPE and mLLDPE, and their melting temperatures are probably too low to influence the tangent line, which is used to obtain the onset temperature.

The hot tack strength is relevant for sealing through solid particles as these particle can push the seal layers away from each other directly after opening the hot jaws when the seal is still hot. ${ }^{15}$ This spring back effect is similar when wrinkles are present. The effect is discussed in several papers. ${ }^{6,7,9,10}$ Figure 3 shows the hot tack results of all films. The hot tack initiation temperature (temperature where a low but measurable hot tack strength is obtained), peak value, and window (temperature range where a relatively high hot tack strength is obtained) are discussed to compare the three packaging films. As there is currently no clear definition of the hot tack initiation temperature, it is defined in this study as the minimum temperature $\left({ }^{\circ} \mathrm{C}\right)$ where a seal with low-hot tack strength is produced, a threshold value of $0.03 \mathrm{~N} \mathrm{~mm}^{-1}$ must be exceeded. The hot tack window was defined as the temperature range $\left({ }^{\circ} \mathrm{C}\right)$ from minimum to maximum temperature where seals with medium hot tack strength are produced, a threshold value of $0.1 \mathrm{~N} \mathrm{~mm}^{-1}$ must be exceeded.

Film 1 has a relatively high hot tack initiation temperature $\left(105^{\circ} \mathrm{C}\right)$ compared with the other films. This can be a result of the absence of low melting main components such as plastomer or sodium ionomer in the lower $15 \mu \mathrm{m}$ of the seal layer. The peak value $\left(0.29 \pm 0.01 \mathrm{~N} \mathrm{~mm}^{-1}\right)$ is low compared with other films. The peak value is reached at $110^{\circ} \mathrm{C}$, which is in accordance with the melting peak temperature of film 1 and the individual granulates of the two main components, LDPE, and mLLDPE. The hot tack window is narrow $\left(110^{\circ} \mathrm{C}-140^{\circ} \mathrm{C}\right)$ compared with the other films. Film 2 has a relative low hot tack initiation temperature $\left(90^{\circ} \mathrm{C}\right)$ making it suitable for high speed sealing applications. This can be a result of the presence of plastomer ${ }^{6}$ in the lower $15 \mu \mathrm{m}$ of the seal layer. Compared with film 1 , the peak value $\left(0.43 \pm 0.03 \mathrm{~N} \mathrm{~mm}^{-1}\right)$ is high, suggesting more and/or deeper entanglement, this was previously described in literature. ${ }^{9}$ For

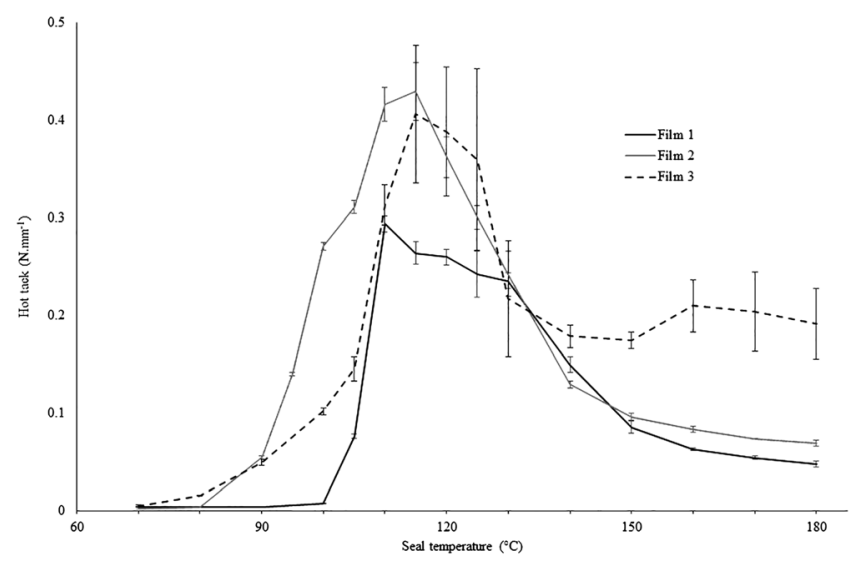

FIGURE 3 Hot tack graph with variation in seal temperature, seal parameters: $1.0 \mathrm{~s}$ seal time- $0.3 \mathrm{~N} \mathrm{~mm}^{-2}$ seal pressure- $0.1 \mathrm{~s}$ cool time $(n=3)$ for three packaging films both films, the hot tack strength decreases strongly in a similar way at elevated temperatures (greater than or equal to $150^{\circ} \mathrm{C}$ ). Film 3, with the sodium ionomer seal layer, shows a larger standard deviation compared with the other films. It has a low hot tack initiation temperature $\left(90^{\circ} \mathrm{C}\right)$, then the hot tack strength slowly increases until a high peak value $\left(0.41 \pm 0.07 \mathrm{~N} \mathrm{~mm}^{-1}\right)$ is reached at $115^{\circ} \mathrm{C}$. The hot tack window is very broad $\left(100^{\circ} \mathrm{C}\right.$-greater than or equal to $\left.180^{\circ} \mathrm{C}\right)$, indicating that this film keeps a large portion of its strength at seal temperatures greater than or equal to $150^{\circ} \mathrm{C}$. The superior hot tack performance of ionomers (high hot tack strength at low seal times and under a wide range of seal temperatures) was previously described in literature. ${ }^{16}$ Both films 2 and 3 are evaluated as good hot tack performers because of a combination of hot tack properties (low initiation temperature, high peak value, and wide window).

\subsection{Evaluation and optimization of contaminated seal strength}

The experimental design of the three films of this study is shown in Table 3. At each of the parameter combinations defined by the design, both clean and contaminated (coffee and blood powder) seals were created, and their seal strength was measured. Other responses such as leak tightness, seal energy, optical aspects, and seal thickness are also possible in a single or multi-response model but were not considered in this study. All clean and contaminated seal strengths of films 1 , 2 , and 3 , produced at the 20 parameter combinations (temperature, time and pressure) defined by the experimental design are also shown in the table. These seal strengths served as an input to build a model that predicts the clean and contaminated seal strength at all possible parameter settings within the defined design space.

In Tables 4, 5, and 6, the coefficients of the terms included in the models for each film are summarized for clean seals, coffee contaminated, and blood powder contaminated seals, respectively. Nonsignificant terms were not included in the model, and therefore no coefficient is shown in the table. Based on these models, the settings of temperature, time, and pressure resulting in maximum seal strength were determined for each film-contaminant combination as described in the Methods section.

As a validation of the optima of the three films, the predicted optimal parameter settings, predicted maximum seal strengths, and limits of the confidence intervals calculated based on the validation experiments (CICon approach) are shown in Table 7.

The predicted values are a good indication of the measured values, but the model tends to slightly overestimate the optimized seal strength. A higher accuracy could be reached by adding repetitions to the test or augmenting the experimental design with additional experiments. This can be a subject for further research. Contamination decreases the seal strength, even when optimized. The rate of decrease is dependent on the used seal material (blend LDPE/mLLDPE, blend LDPE/plastomer, and sodium ionomer) and the applied contamination (ground coffee and blood powder). For films 1,2 , and 3 , the degrees of decrease, based on the measured average values (not shown in table), are, respectively, $25 \%, 16 \%$, and $63 \%$ for 
TABLE 3 Experimental design with parameters (seal temperature [T], time [t], and pressure [p]) and responses (clean and contaminated seal strength) of films 1, 2, and 3 (F1, F2, and F3)

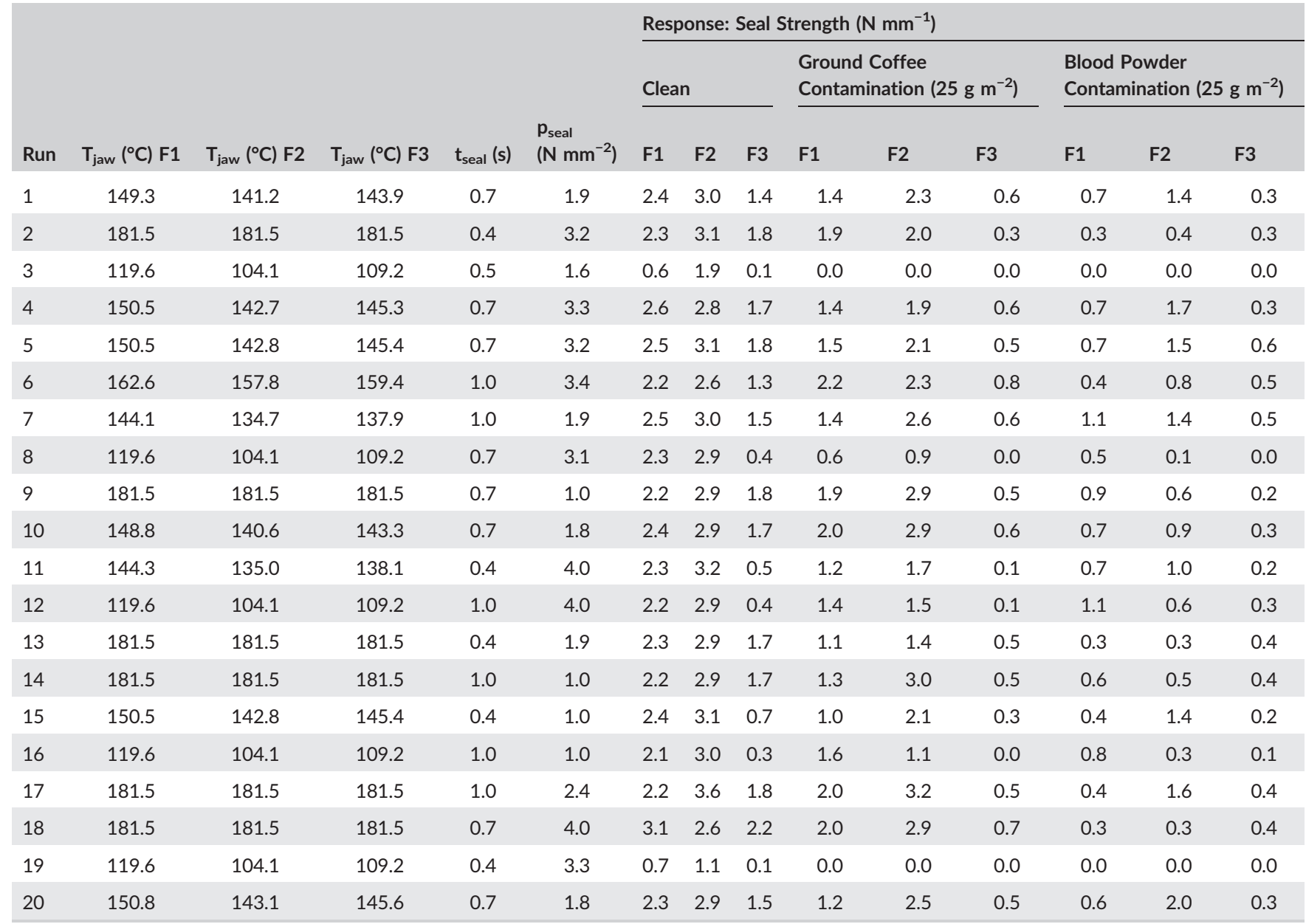

TABLE 4 Significant coefficients, terms, and regression significance of the seal strength model for clean seals of films 1, 2, and 3

\begin{tabular}{|c|c|c|c|c|c|c|c|}
\hline \multirow[b]{2}{*}{ Coefficient } & \multirow[b]{2}{*}{ Term } & \multicolumn{2}{|l|}{ Film 1} & \multicolumn{2}{|l|}{ Film 2} & \multicolumn{2}{|l|}{ Film 3} \\
\hline & & Value & $P$ value & Value & $P$ value & Value & $P$ value \\
\hline$\beta_{0}$ & Intercept & -0.2435 & .6765 & 1.5979 & .0314 & -2.0659 & .0001 \\
\hline$\beta_{2}$ & $\mathrm{t}$ & 0.7350 & .0295 & 0.6962 & .1144 & 0.5145 & .0364 \\
\hline$\beta_{3}$ & $\mathrm{p}$ & 0.0981 & .1577 & -0.0479 & .6028 & 0.0552 & .2711 \\
\hline$\beta_{23}$ & $t^{*} p$ & - & - & -0.1641 & .6741 & - & - \\
\hline$\beta_{13}$ & $T^{*} p$ & - & - & -0.0012 & .7122 & - & - \\
\hline$\beta_{11}$ & $\mathrm{~T}^{2}$ & -0.0005 & .0089 & -0.0003 & .0857 & -0.0001 & .1203 \\
\hline$\beta_{22}$ & $t^{2}$ & -2.7627 & .1164 & - & - & -4.1615 & .0046 \\
\hline
\end{tabular}

ground coffee and $71 \%, 45 \%$, and $79 \%$ for blood powder contamination compared with the clean seal strength. The samples contaminated with coffee reach a higher optimized seal strength than the samples with blood powder. This can be a result of more binding spots between the seal layers because of the lower amount of coffee particles when a same mass of contamination is applied. Figure 4 shows that there are more clean areas with contaminated seal samples with coffee particles compared with those with blood powder.

Seal pressure has a slight or no influence on the seal strength as shown in Tables 4, 5, and 6. There is no significant effect of pressure 
TABLE 5 Significant coefficients, terms, and regression significance of the seal strength model for coffee contaminated seals of films 1,2 , and 3

\begin{tabular}{|c|c|c|c|c|c|c|c|}
\hline \multirow[b]{2}{*}{ Coefficient } & \multirow[b]{2}{*}{ Term } & \multicolumn{2}{|l|}{ Film 1} & \multicolumn{2}{|l|}{ Film 2} & \multicolumn{2}{|l|}{ Film 3} \\
\hline & & Value & $P$ value & Value & $P$ value & Value & $P$ value \\
\hline$\beta_{0}$ & Intercept & -1.0186 & .0085 & -1.8826 & .0177 & -0.7098 & .0111 \\
\hline$\beta_{2}$ & $\mathrm{t}$ & 1.2563 & $<.0001$ & 1.0453 & .0104 & 0.0202 & .8879 \\
\hline$\beta_{3}$ & $p$ & 0.0893 & .0347 & -0.0446 & .8187 & -0.0254 & .4254 \\
\hline$\beta_{23}$ & $t^{*} p$ & -0.3708 & .0362 & - & - & - & - \\
\hline$\beta_{13}$ & $T^{*} p$ & - & - & - & - & -0.0029 & .0232 \\
\hline$\beta_{11}$ & $\mathrm{~T}^{2}$ & -0.0006 & $<.0001$ & -0.0006 & .0004 & -0.0001 & .1426 \\
\hline$\beta_{22}$ & $t^{2}$ & - & - & - & - & - & - \\
\hline
\end{tabular}

TABLE 6 Significant coefficients, terms, and regression significance of the seal strength model for blood powder contaminated seals of films 1, 2, and 3

\begin{tabular}{|c|c|c|c|c|c|c|c|}
\hline \multirow[b]{2}{*}{ Coefficient } & \multirow[b]{2}{*}{ Term } & \multicolumn{2}{|l|}{ Film 1} & \multicolumn{2}{|l|}{ Film 2} & \multicolumn{2}{|l|}{ Film 3} \\
\hline & & Value & $P$ value & Value & $P$ value & Value & $P$ value \\
\hline$\beta_{0}$ & Intercept & 0.4262 & .1593 & 0.5214 & .3284 & -0.5112 & .0049 \\
\hline$\beta_{2}$ & $\mathrm{t}$ & 0.6953 & .0006 & 0.5969 & .1189 & 0.3370 & .0025 \\
\hline$\beta_{3}$ & $\mathrm{p}$ & -0.0194 & .5617 & - & - & 0.0404 & .0582 \\
\hline$\beta_{23}$ & $t^{*} p$ & -0.2909 & .0587 & - & - & - & - \\
\hline$\beta_{13}$ & $T^{*} p$ & -0.0047 & .0059 & - & - & - & - \\
\hline$\beta_{11}$ & $\mathrm{~T}^{2}$ & -0.0003 & .0013 & -0.0007 & $<.0001$ & -0.0001 & .0042 \\
\hline$\beta_{22}$ & $t^{2}$ & - & - & - & - & 0.4579 & .3619 \\
\hline
\end{tabular}

TABLE 7 Validation of statistical optimum $(n=10)+$ optimal settings

\begin{tabular}{|c|c|c|c|c|c|c|}
\hline & \multicolumn{6}{|c|}{ Seal Strength $\left(\mathrm{N} \mathrm{mm}^{-1}\right)$} \\
\hline & \multicolumn{2}{|l|}{ Clean } & \multicolumn{2}{|c|}{ Ground Coffee Contamination ( $25 \mathrm{~g} \mathrm{~m}^{-2}$ ) } & \multicolumn{2}{|c|}{ Blood Powder Contamination $\left(25 \mathrm{~g} \mathrm{~m}^{-2}\right)$} \\
\hline & Predicted & $\mathrm{Cl}$ measured & Predicted & $\mathrm{Cl}$ measured & Predicted & $\mathrm{Cl}$ measured \\
\hline Film 1 & 3.0 & $2.2-2.7$ & 2.1 & $1.6-2.0$ & 1.1 & $0.6-0.9$ \\
\hline Film 2 & 3.3 & $3.0-3.2$ & 3.1 & $2.3-2.8$ & 1.6 & 1.4-1.9 \\
\hline Film 3 & 2.2 & $1.7-2.0$ & 0.9 & $0.6-0.8$ & 0.5 & $0.3-0.5$ \\
\hline Film 1 & \multicolumn{2}{|c|}{$165^{\circ} \mathrm{C}_{-} 0.7 \mathrm{~s} \_4.0 \mathrm{~N} \mathrm{~mm}^{-2}$} & \multicolumn{2}{|c|}{$151^{\circ} \mathrm{C}_{-} 1.0 \mathrm{~s} \_1.0 \mathrm{~N} \mathrm{~mm}^{-2}$} & \multicolumn{2}{|c|}{$150^{\circ} \mathrm{C} \_1.0 \mathrm{~s} \_1.0 \mathrm{~N} \mathrm{~mm}^{-2}$} \\
\hline Film 2 & \multicolumn{2}{|c|}{$144^{\circ} \mathrm{C} \_1.0 \mathrm{~s} \_1.0 \mathrm{~N} \mathrm{~mm}^{-2}$} & \multicolumn{2}{|c|}{$161^{\circ} \mathrm{C}_{-} 1.0 \mathrm{~s} \_4.0 \mathrm{~N} \mathrm{~mm}^{-2}$} & \multicolumn{2}{|c|}{$147^{\circ} \mathrm{C} \_1.0 \mathrm{~s} \_2.0 \mathrm{~N} \mathrm{~mm}^{-2}$} \\
\hline Film 3 & \multicolumn{2}{|c|}{$182^{\circ} \mathrm{C}_{-} 0.7 \mathrm{~s} \_2.7 \mathrm{~N} \mathrm{~mm}^{-2}$} & \multicolumn{2}{|c|}{$182^{\circ} \mathrm{C}_{-} 0.4 \mathrm{~s} \_1.2 \mathrm{~N} \mathrm{~mm}^{-2}$} & \multicolumn{2}{|c|}{$157^{\circ} \mathrm{C}_{-} 1.0 \mathrm{~s} \_3.4 \mathrm{~N} \mathrm{~mm}^{-2}$} \\
\hline
\end{tabular}

Abbreviation: $\mathrm{Cl}$, confidence interval. 
(A)

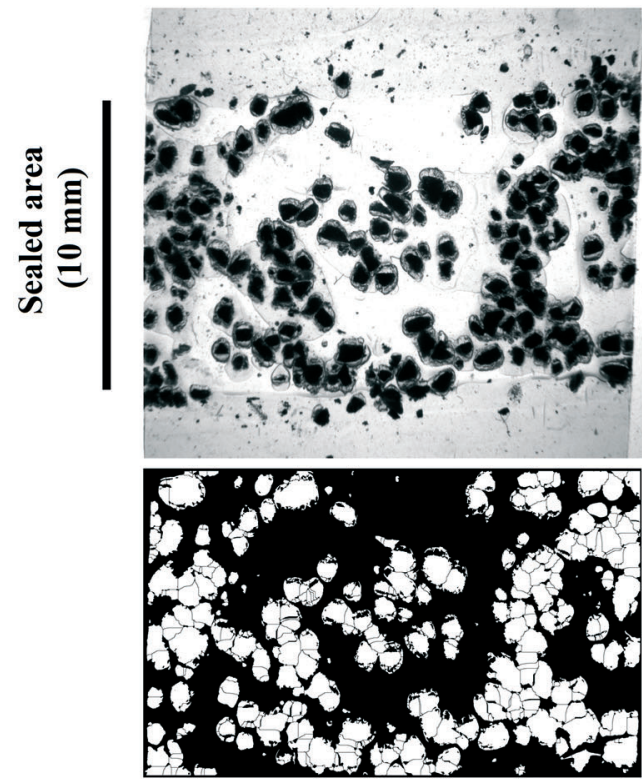

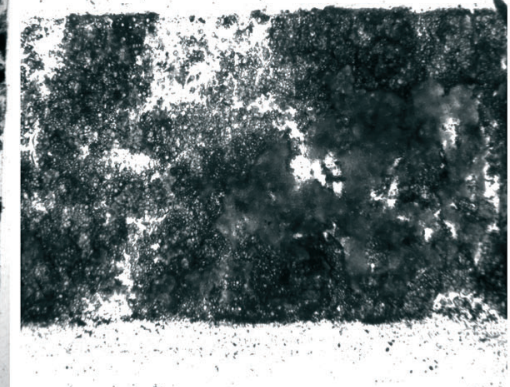

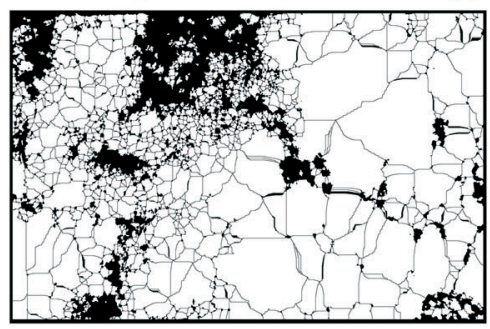

FIGURE 4 A, Raw images of coffee (left) and blood powder (right) contaminated samples of film 2, sealed at $181.5^{\circ} \mathrm{C}, 0.7 \mathrm{~s}$, and $1.0 \mathrm{~N} \mathrm{~mm}{ }^{-2}$ with a high resolution digital imaging set up with LED backlight illumination for high contrast. These raw images are converted to B, binary images where clean areas are black

on seal strength with clean seals. Previous research on clean seals described the very limited influence of seal pressure on seal strength. ${ }^{17,18}$ With ground coffee contamination, a slight effect of pressure is seen as the first order and $t^{*} p$ term of film 1 and T*p term of film 3 have significant effects. With blood powder contamination, there is only a significant effect of the $T^{*} p$ term on seal strength for film 1. Higher significance is observed for temperature and time and the combination of both parameters on clean and contaminated seal strength. This result is in line with previous studies that state that temperature and time are the most important factors influencing seal strength. ${ }^{17,18}$ These parameters were used in process windows within which at least $90 \%$ of the maximum seal strength is obtained. The process windows for the three films are shown in Figure 5. Films 1 and 2 have the widest process window when seals are clean, and process windows become narrow when solid contamination is present. Process windows for clean and coffee contaminated seals are wider for film 2 than for the other films. Even at low seal times of 0.5 to $0.6 \mathrm{~s}$, it is possible to produce strong seals if the temperature is set at $170^{\circ} \mathrm{C}$. In an industrial context, this is an advantageous film property with respect to production speed. Blood powder contaminated seals need higher seal time to produce strong seals. Taken into account the optimal values of Table 7 and the process windows of Figure 4, film 1 (seal layer blend of LDPE and mLLDPE) is less tolerant for solid contamination than film 2 (seal layer blend of LDPE and plastomer) regarding seal strength. The results of this comparison are in line with the comparison of hot tack performance between both films (lower initiation, wider window, and higher peak value for film 2). For film 3, all process windows are narrow compared with films 1 and 2. There is almost no overlap between the process windows. Taken into account the seal strengths of Table 7 and the process windows of Figure 5, this film has the worst tolerance for these types of solid contamination regarding seal strength. These results are inconsistent with the good hot tack performance (low seal initiation, high peak value, and wide window) of this film. One possible explanation would be that for this film, only the lower part of the seal layer participates in the encapsulation of the particles. The lower thickness (5- $\mu \mathrm{m}$ acid copolymer $+10-\mu \mathrm{m}$ ionomer) of the effective seal layer compared with the particle size could decrease the seal through contamination performance. Another possible explanation can lie within the flow behaviour of the seal material. In previous research, ${ }^{9}$ flow ability was related to the encapsulation of milk powder particles. These particles can be isolated if the seal material can flow around them. Both topics can be interesting for further research to gain better insight into the clean and contaminated seal performance of packaging films.

All samples that were optimized in seal strength are tested for their leak tightness by a dye penetration test prior to the seal strength test. Samples are tested in tenfold. All clean optimized samples were leak tight. In the case of coffee contamination, films 1 and 3 have, respectively, 8 and 7 out of 10 leak tight samples. All samples of film 2 were leak tight at optimal settings. In the case of blood powder contamination, film 1 has 9 out of 10 leak tight seals, and films 2 and 3 are leak tight at the optimal settings. Comparing the three seal layers, the plastomer-based seal layer in film 2 has the best seal through contamination performance regarding leak tightness at optimal settings. A previous study ${ }^{15}$ suggests that viscous, hot tack, and mechanical properties of seal materials are related with the encapsulation of solid contamination. Low zero shear viscosity, a high hot tack strength window (=area under hot tack curve between hot tack initiation temperature and actual seal jaw temperature), and high resistance to elongation under stress were beneficial for preventing leaks. ${ }^{15}$ 
Film 1

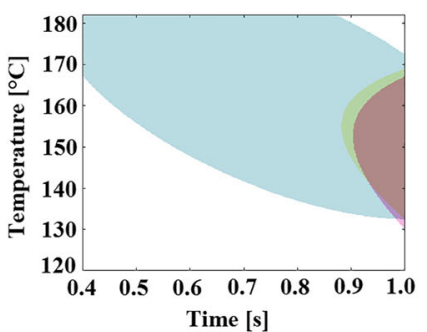

Film 2

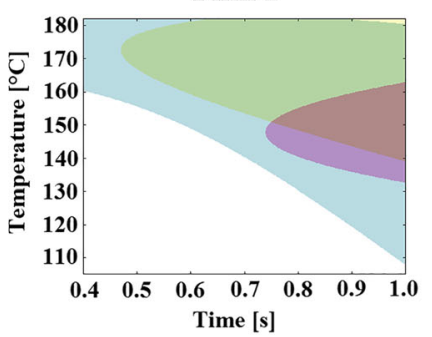

Film 3

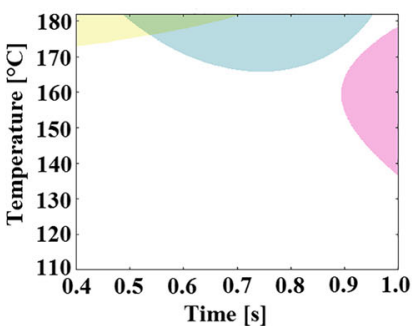

$\square$ Clean $\square$ Coffee $\square$ Blood powder

FIGURE 5 Temperature vs time process windows for clean, coffee, and blood powder contaminated samples. The process windows indicate those combinations of temperature and time that result in a seal strength of at least $90 \%$ of the optimum value. Pressure was kept constant at $2.5 \mathrm{~N} \mathrm{~mm}^{-2}$

\section{5 | CONCLUSIONS}

In this study, a method to optimize the granular contaminated seal strength of packaging films is presented. The optimal values predicted by the response surface method are experimentally validated. Predicted values are a good indication of clean and contaminated seal strength, although there is a tendency of overestimation by the model. Augmenting the initial experimental design or including repetitions in the design could improve this. Besides giving optimal values at one specific parameter setting, process windows for clean and contaminated seals can be obtained by doing a limited amount of tests. These process windows are highly relevant for practical use in industry. Solid contamination causes a decrease in the maximal seal strength and narrows down the parameter region of time and temperature in the process window where $90 \%$ of that maximal strength is obtained compared with clean seals. When an equal mass of coffee and blood powder is applied, blood powder has a more negative impact on the maximum strength than coffee powder.

The influence of variation in seal layer composition on the seal through contamination performance of packaging films is evaluated. The film with the plastomer-based seal layer outperformed the other films with a higher seal strength, wider process windows, and a higher degree of leak tightness (evaluated with the dye penetration test). This film also has a better clean seal performance than the other ones. Regarding the use of hot tack as a predictive test for the contaminated seal strength, it can be concluded that there are similarities in the comparison of films with metallocene and plastomer-based seal layer, such as the hot tack initiation temperature, window, and peak value. The hot tack results of the film with sodium ionomer, however, were not predictive for the contaminated seal strength.

The influence of seal technology, jaw geometry, and effective thickness and flow behaviour of the seal layer on contaminated seal performance can be subjects of further research.

\section{ACKNOWLEDGEMENTS}

This research was performed within the CORNET project "EVOCOSEAL: Evaluation and Optimization of Contaminated Seal Performance for Food Packaging," funded by the Flemish (Agentschap
Innoveren \& Ondernemen (VLAIO-TETRA nr. 150817)) and German government (German Federal Ministry for Economic Affairs and Energy (BMWi, IGF project no. 172 EBR)).

\section{ORCID}

Bram Bamps (D) https://orcid.org/0000-0002-8992-5098

\section{REFERENCES}

1. Hishinuma K. Heat Sealing Technology And Engineering For Packaging: Principles And Applications. Lancaster: DEStech Publications inc; 2009:30-42 ISBN: 978-1-932078-85-5.

2. Tauschitz B, Washüttl M, Wepner B, Tacker M. MAP-Verpackungen: ein Drittel nicht optimal. PACKaktuell, DE. 2003;04:6-8.

3. Dudbridge M, Turner R. Seal integrity and the impact on food waste. http://www.wrap.org.uk/sites/files/wrap/Household_food_and_drink_ waste_in_the_UK_-_report.pdf, date of access:22/11/2018. WRAP 2009. ISBN: 1-84405-430-6.

4. Morris B. The Science And Technology Of Flexible Packaging: Multilayer Films From Resin And Process To End Use. Amsterdam: Elsevier inc 2016; 2017:216-218 249-250. ISBN: 978-0-323-24273-8.

5. Simpson MF, Presa JL. (1996): A comparison of AFFINITY plastomers produced via INSITE Technology with ethylene/acrylic acid copolymers, ionomers, and ULDPE. In: Future-Pak 1996, Chicago, USA.

6. Mesnil P, Rohse N, Arnauts J. Halle, RW. (2000): Seal trough contamination performance of metallocene plastomers. In: TAPPI polymers, laminations, \& coatings conference 2000 , Chicago, USA.

7. Morris B. Sure ways to reduce package sealing failure. http://www2. dupont.com/Packaging_Resins/en_US/assets/downloads/white_ papers/Reduce_Package_Sealing_Failure_Barry_Morris_November_ 2010.pdf, date of access: 22/11/2018. Dupont 2010.

8. Bach S, Thürling K, Majschak J-P. Ultrasonic sealing of flexible packaging films - principle and characteristics of an alternative sealing method. Packag Technol Sci. 2011;25(4):233-248. https://doi.org/10.1002/ pts.972

9. Sadegi F, Ajji A. Application of single site catalyst metallocene polyethylenes in extruded films: effect of molecular structure on sealability, flexural cracking and mechanical properties. Can J Chem Eng 2014. 2014;92(7):1181-1188. https://doi.org/10.1002/cjce.21964

10. Bilgen M, Van Dun J. Hermetic sealing of flexible packages. In: International polyolefins conference 2014, Houston, USA.

11. D'huys, K, Bamps B., Peeters R, De Ketelaere B. Multi-criteria evaluation and optimization of the ultrasonic sealing performance based on 
design of experiments and response surface methodology. Accepted for publication in packaging technology and science.

12. Antony J. Design of Experiments for Engineers and Scientists. Amsterdam: Elsevier; 2003 ISBN: 9780750647090.

13. Jensen WA. Confirmation runs in design of experiments. J Qual Technol. 2016;48(2):162-177. https://doi.org/10.1080/00224065.2016.11918157

14. Sierra JD, Noriega MD, Nicolais V. Effect of metallocene polyethylene on heat sealing properties of low density polyethylene blends. J Plast Film Sheeting. 2000;16(1):33-42. https://doi.org/10.1106/YYFG9KH1-R7QU-VK9H

15. Ward M, Li M. Seal-through-contamination and caulkability-an evaluation of sealants' ability to encapsulate contaminants in the seal area. In: Tappi place conference 2016, Fort Worth, USA.

16. Suenaga S, Hirasawa E. Connection between hot tack force and formfill-seal performance of packaging films. JSTD. 2004;16(7):450-458. https://doi.org/10.4325/seikeikakou.16.450
17. Theller HW. Heatsealability of flexible web materials in hot-bar sealing applications. J Plast Film Sheeting. 1989;5(1):66-93. https://doi.org/ 10.1177/875608798900500107

18. Stehling FC, Meka P. Heat sealing of semicrystalline polymer films. I. Calculation and measurement of interfacial temperatures: effect of process variables on seal properties. J Appl Polym Sci. 1994;51(1):89-103. https://doi.org/10.1002/app.1994.070510111

How to cite this article: Bamps B, D'huys K, Schreib I, Stephan B, De Ketelaere B, Peeters R. Evaluation and optimization of seal behaviour through solid contamination of heatsealed films. Packag Technol Sci. 2019;1-10. https://doi.org/ 10.1002/pts.2442 